\title{
Diaspirin Cross-Linked Hemoglobin Fails to Improve Left Ventricular Diastolic Function after Fluid Resuscitation from Hemorrhagic Shock
}

\author{
A. Pape ${ }^{a} \quad$ G. Kemming ${ }^{a}$ b $\quad$ F. Meisner ${ }^{b} \quad$ M. Kleen ${ }^{a}$ O. Hablera \\ ${ }^{a}$ Clinic of Anaesthesiology and bInstitute for Surgical Research, Ludwig Maximilians University, Munich, Germany
}

\section{Key Words}

Diaspirin cross-linked hemoglobin - Hemorrhagic shock • Coronary stenosis · Diastolic function

\begin{abstract}
In severe hemorrhagic shock, left ventricular (LV) diastolic dysfunction is an early sign of cardiac failure due to compromised myocardial oxygenation. Immediate fluid replacement or, in particular, administration of a hemoglobin-based oxygen carrier (diaspirin cross-linked hemoglobin; DCLHb) improves myocardial oxygenation; therefore, positive effects on LV diastolic function could be expected. The effects of fluid resuscitation from severe hemorrhagic shock with $\mathrm{DCLHb}$ were investigated in 20 anesthetized domestic pigs. After generation of a critical left anterior descending coronary artery stenosis (narrowing of the artery until disappearance of reactive hyperemia after a 10-second complete vessel occlusion), hemorrhagic shock (mean arterial blood pressure $45 \mathrm{~mm}$ $\mathrm{Hg}$ ) was induced within $15 \mathrm{~min}$ by controlled blood withdrawal and maintained for 60 min. Fluid resuscitation consisted of replacement of the plasma volume withdrawn during hemorrhage by infusion of either $10 \%$ DCLHb (DCLHb group, $n=10$ ) or $8 \%$ human serum albumin (HSA) oncotically matched to DCLHb (HSA group,
\end{abstract}

\section{KARGER}

Fax +41613061234

E-Mail karger@karger.ch

www. karger.com
(C) 2001 S. Karger AG, Basel

0014-312X/01/0336-0318\$17.50/0

Accessible online at:

www.karger.com/journals/esr $n=10)$. After completion of resuscitation, an observation period of $60 \mathrm{~min}$ elapsed. Measurements of central hemodynamics, myocardial oxygenation, and LV diastolic function were performed at baseline, after induction of critical coronary artery stenosis, after $60 \mathrm{~min}$ of hemorrhagic shock, immediately after resuscitation, and 60 min later. While 5 out of 10 animals treated with HSA died within the first 20 min after fluid resuscitation from acute LV pump failure, all DCLHb-treated animals survived until the end of the protocol $(p<0.05)$. Despite superior myocardial oxygenation due to augmentation of the arterial $\mathrm{O}_{2}$ content as well as of coronary perfusion pressure, no beneficial effects on LV diastolic function were observed after infusion of DCLHb. Peak velocity of LV pressure decrease $\left(\mathrm{dp} / \mathrm{dt}_{\mathrm{min}}\right)$ did not reveal significant differences between the two groups. Immediately after completion of fluid resuscitation with DCLHb, the time constant of LV diastolic relaxation $(\tau)$ was prolonged when compared with HSA-treated animals ( $p<0.05$ ), indicating retardation of early LV diastolic relaxation. Our data suggest that DCLHb fails to improve LV diastolic function after fluid resuscitation from severe hemorrhagic shock. However, positive effects on myocardial perfusion and oxygenation result in a significant reduction of the mortality of severe hemorrhagic shock.

Andreas Pape, MD

Clinic of Anaesthesiology, Ludwig Maximilians University

Nussbaumstrasse 20, D-80336 Munich (Germany)

Tel. +498951602691, Fax +498951604446

E-Mail andreas.pape@helios.med.uni-muenchen.de 


\section{Introduction}

Adequate cardiac performance is crucial for the outcome after fluid resuscitation from severe hemorrhagic shock, since heart failure induced by dilutional anemia may become the life-limiting complication in this situation $[1,2]$. An early marker of left ventricular (LV) dysfunction despite augmented systolic contractility is the alteration of LV diastolic properties during shock [3].

The LV diastolic function strictly depends on an adequate oxygenation of the myocardium [4]. During shock, the latter is jeopardized by a significant reduction of the coronary perfusion pressure (CPP) and thus myocardial perfusion.

Since primary therapy of hemorrhagic shock is usually performed with acellular, i.e., crystalloidal and colloidal solutions, the arterial $\mathrm{O}_{2}$ content falls due to the dilution of the remaining red blood cell (RBC) mass. Depending on the degree of dilution, myocardial oxygenation may become critical, a reason why fluid resuscitation with cellfree solutions was found ineffective in restoration of myocardial $\mathrm{O}_{2}$ supply $[5,6]$. Reduction of myocardial $\mathrm{O}_{2}$ supply enhances myocardial stiffness [4] as an early sign of LV diastolic dysfunction.

Actually, the 'state-of-the art' therapy to overcome the reduced myocardial $\mathrm{O}_{2}$ supply is the transfusion of packed RBC. An alternative to this RBC transfusion might be the immediate infusion of a hemoglobin-based artificial oxygen carrier (HBOC) [7, 8]. HOBCs are hyperoncotic solutions acting like 'plasma expanders' with the ability to transport $\mathrm{O}_{2}$ [9]. Therefore, most of the HBOCs seem to be suitable for the initial treatment of hemorrhagic shock. Moreover, most of the HBOCs possess specific vasoconstrictive properties, mainly induced by scavenging of the endogeneic vasodilator nitric oxide $[10,11]$.

In the present experimental animal model, the effects on LV diastolic function were investigated after infusion of $10 \%$ diaspirin cross-linked hemoglobin (DCLHb) and compared to an equioncotic human serum albumin (HSA) solution. In all animals the LV performance was initially compromised by a critical stenosis of the left anterior descending artery (LAD) [12]. With respect to the vasoconstrictive and $\mathrm{O}_{2}$ transport properties of DCLHb, we assumed positive effects on the LV diastolic function, leading to the hypothesis that fluid resuscitation from hemorrhagic shock with DCLHb would (1) improve LV diastolic function and (2) prove superior to HSA void of $\mathrm{O}_{2}$-transporting and vasoconstrictive properties.

\section{Materials and Methods}

The experiments were performed as part of a comprehensive experimental program; parts of the data have been published already [see 6]. The experimental protocol has been described in detail; therefore, in the following, we concentrate on the methodology exerted for analysis of the diastolic function.

\section{Anesthesia and Animal Preparation}

20 healthy farm-bred pigs of either sex (body weight $26.2 \pm$ $4.2 \mathrm{~kg}$ ) were anesthetized by intramuscular injection of $10 \mathrm{mg} / \mathrm{kg}$ ketamine, $1 \mathrm{mg} / \mathrm{kg}$ midazolam, and $0.03 \mathrm{mg} / \mathrm{kg}$ atropine, followed by intravenous injection of $1.5 \mathrm{mg} / \mathrm{kg}$ methohexital, $0.03 \mathrm{mg} / \mathrm{kg}$ fentanyl, and $0.2 \mathrm{mg} / \mathrm{kg}$ pancuronium and by continuous intravenous infusion of midazolam $(0.02 \mathrm{mg} / \mathrm{kg} / \mathrm{min})$, fentanyl $(0.8 \mu \mathrm{g} / \mathrm{kg} / \mathrm{min})$, and pancuronium $(4 \mathrm{mg} / \mathrm{h}$; for trade names of applied anesthetics $\mathrm{cf}$. appendix). Ventilation with $\mathrm{FiO}_{2}=0.5$ was adjusted to maintain arterial normocapnia. The animals were instrumented with two tip manometer catheters (PC 370; Millar Instruments, Houston, Tex., USA) placed into the abdominal aorta and into the chamber of the left ventricle, respectively, and a Swan-Ganz catheter (right ventricular ejection fraction/volumetric catheter; Baxter, Irvine, Calif., USA) floated into the pulmonary artery. After midline sternotomy, the pericardium was opened, the LAD dissected free from the surrounding tissue, and two Teflon-coated copper-wires were wound around the LAD for induction of critical coronary stenosis. Two pairs of piezoelectronic ultrasonic transducers $(5 \mathrm{MHz}$, diameter $1.5 \mathrm{~mm}$; Dr. W. Heimisch, Deutsches Herzzentrum, Munich, Germany) were inserted into the subendocardial layer of the LV myocardium, one pair being located in the anterior and one in the posterior wall.

\section{Experimental Protocol}

After termination of surgical preparation and placement of the different measuring devices, a 15-min stabilization period was allowed to elapse, and the first set of measurements ('baseline') was performed. According to the definition of Gould et al. [13], a critical coronary stenosis (tightening the LAD until loss of reactive hyperemia after a 10-second complete vessel occlusion) was induced, followed by the second measurement ('critical stenosis'). Hemorrhagic shock (mean aortic pressure, MAP, $45 \mathrm{~mm} \mathrm{Hg}$ ) was induced within $15 \mathrm{~min}$ by controlled withdrawal of blood and maintained for $60 \mathrm{~min}$ by further withdrawal or uptake of blood (third measurement: '60 min shock') [14]. Thereafter, the animals were resuscitated according to a prospectively fixed randomization plan with either $10 \%$ DCLHb or $8 \%$ HSA (both provided by Baxter Healthcare, Deerfield, Ill., USA) oncotically matched to DCLHb (infusion rate $2 \mathrm{ml} / \mathrm{kg} / \mathrm{min}$; table 1). The infused volume of either solution was equivalent to the volume of plasma withdrawn during induction of shock ( $16 \pm 3 \mathrm{ml} \mathrm{DCHLb}$, $18 \pm 3 \mathrm{ml} \mathrm{HSA}$ ). Immediately after completion of fluid resuscitation, the fourth measurement ('resuscitation') was performed, followed by an observation period of $60 \mathrm{~min}$ and the final measurement ('60 min resuscitation'). Finally, all animals were sacrificed by intracardial injection of a saturated potassium chloride solution.

\section{Parameters}

Hemodynamics. Heart rate, MAP, LV pressure (LVP), LV enddiastolic pressure (LVEDP), central venous pressure (CVP), and mean pulmonary arterial pressure were determined with the devices described above. The systemic vascular resistance was calculated and indexed to body surface area (cf. appendix). 


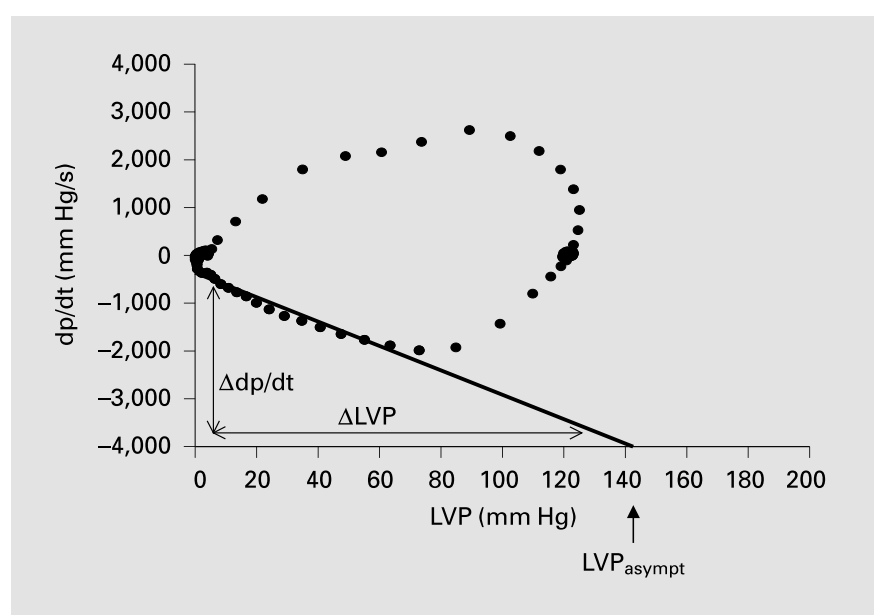

Fig. 1. Determination of the time constant of LV diastolic relaxation $(\tau)$ as the negative reciprocal of the asymptotic slope between $\mathrm{LVP}_{0}$ and $\mathrm{LVP}_{\text {asympt }}$.

Table 1. Characteristics of the two resuscitation fluids investigated

\begin{tabular}{lll}
\hline Parameter & $10 \%$ DCLHb & $8 \%$ Albumin \\
\hline $\mathrm{pH}$ at $37^{\circ} \mathrm{C}$ & 7.4 & 7.41 \\
Colloid osmotic pressure, $\mathrm{mm} \mathrm{Hg}$ & 42 & 43.8 \\
Endotoxin activity, EU/ml & $<0.1$ & $<0.06$ \\
Osmolality, mosm/kg & 300 & 284 \\
Sodium, $\mathrm{mEq} / \mathrm{l}$ & 140 & 132 \\
Potassium, $\mathrm{mEq} / \mathrm{l}$ & 4.0 & 3.8 \\
Calcium, $\mathrm{mEq} / \mathrm{l}$ & 2.3 & 1.6 \\
Magnesium, $\mathrm{mEq} / \mathrm{l}$ & 1.0 & 0.7 \\
Chloride, $\mathrm{mEq} / \mathrm{l}$ & 110 & 116 \\
Lactate, $\mathrm{mEq} / \mathrm{l}$ & 32 & 36 \\
\hline
\end{tabular}

Myocardial Oxygenation. Myocardial $\mathrm{O}_{2}$ delivery was calculated as the product of regional myocardial blood flow and arterial $\mathrm{O}_{2}$ content. The CPP was calculated as the difference between diastolic aortic pressure and LVEDP (cf. appendix). The myocardial perfusion was assessed by the radioactive microsphere technique which has already been described in detail elsewhere $[6,15,16]$.

Arterial and coronary venous blood gases were determined with an ABL 300 device (Radiometer, Copenhagen, Denmark). Concentration and $\mathrm{O}_{2}$ saturation of hemoglobin were measured by absorbance spectrophotometry (682 CO oximeter; Instrumentation Laboratory, Lexington, Mass., USA). After infusion of DCLHb, the hemoglobin content in whole blood was the sum of erythrocytic, native hemoglobin and DCLHb dissolved in plasma. Therefore, absorbance was first analyzed in whole blood. Subsequently, blood samples were centrifuged (160 $\mathrm{g}$ for $15 \mathrm{~min}$ ), and the absorbance was determined within the plasma (i.e., the concentration of DCLHb). The $\mathrm{O}_{2}$ saturation kinetics of DCLHb were deduced from a specific $\mathrm{O}_{2}$-binding curve supplied by Baxter Healthcare.
LV Diastolic Function. The global LV compliance was deduced from the maximum rate of $\mathrm{LV}$ pressure decline $\left(\mathrm{LVdp} / \mathrm{dt}_{\mathrm{min}}\right)$. The time constant of LV diastolic pressure decrease ' $\tau$ ' was determined as published by Beattie et al. [17]. The monoexponential pressure-time decay relationship is described as:

$$
\operatorname{LVP}(\mathrm{t})=\left[\mathrm{LVP}_{0}-\mathrm{LVP}_{\text {asympt }}\right] \cdot \mathrm{e}^{\mathrm{At}}+\mathrm{LVP}_{\text {asympt }}
$$

where $\mathrm{A}=$ negative reciprocal of $\tau(\tau=-1 / \mathrm{A}), \mathrm{LVP}_{0}=\mathrm{LVP}$ when $\mathrm{t}=$ 0 , and $\mathrm{LVP}_{\text {asympt }}=$ asymptotic LVP when $\mathrm{t} \rightarrow \infty$ (fig. 1). Mathematical transformation yields:

$$
\mathrm{dp}(\mathrm{t}) / \mathrm{dt}=\mathrm{A} \cdot \operatorname{LVP}(\mathrm{t})-\mathrm{a} \cdot \mathrm{LVP}_{\text {asympt }}
$$

When LVdp/dt is plotted against LVP, linear least-square regression between $\mathrm{LVdp} / \mathrm{dt}_{\min }$ and $\mathrm{LVdp} / \mathrm{dt}=0$ yields a slope of $-1 / \tau$ in milliseconds and the intersection with the abscissa coinciding with $\mathrm{LVP}_{\text {asmypt }}$.

The regional function of the normally perfused and poststenotic myocardium was assessed by sonomicrometric measurement of myocardial segment lengths defined by the distance between the piezoelectronic crystals at end systole and end diastole. End systole was defined as the maximal slope of LVP (i.e., $L V d p / \mathrm{dt}_{\max }$ ), end diastole as the onset of $\mathrm{LV}$ isovolumetric contraction.

\section{Statistics}

Statistical analysis was performed using the SAS 6.12 software package (SAS Institute, Cary, N.C., USA). The distribution of data was tested by the Shapiro-Wilks test. Normally distributed data were analyzed by ANOVA. Significant 'within-group' differences over time detected by ANOVA were further analyzed with a post hoc Student-Newman-Keuls test. Significant 'between-group' differences were further analyzed with a Student $t$ test. Normally distributed parameters are depicted as mean $\pm \mathrm{SD}$. When the data were not normally distributed, an ANOVA on ranks (rANOVA) was performed. Post hoc analysis of differences revealed by rANOVA was performed with a Wilcoxon signed-rank test. These results are depicted as median \pm semi-interquartile range. The alpha error was set at $5 \%$ for either test.

\section{Results}

Parts of the results of this extensive study have already been published $[6,16]$. Briefly, it was demonstrated that all animals treated with DCLHb survived the observation period after completion of fluid resuscitation, whereas 5 out of 10 HSA-treated animals died of acute LV failure within $20 \mathrm{~min}$ after termination of resuscitation. This finding was explained by superior myocardial oxygenation after infusion of DCLHb, resulting from augmentation of $\mathrm{CaO}_{2}$ as well as from restoration of CPP, leading to complete reversal of shock-induced subendocardial ischemia and to improvement of shock-induced disturbances of the distribution of regional myocardial perfusion. Moreover, it could be shown that $60 \mathrm{~min}$ after resuscitation, $33 \%$ of the $\mathrm{O}_{2}$ consumed by the poststenotic myocardium was delivered by DCLHb. 
Table 2. Hemodynamics and arterial $\mathrm{O}_{2}$-content

\begin{tabular}{|c|c|c|c|c|c|c|}
\hline Parameter & Group & Baseline & $\begin{array}{l}\text { Critical } \\
\text { coronary stenosis }\end{array}$ & $\begin{array}{l}60 \mathrm{~min} \\
\text { shock }\end{array}$ & Resuscitation & $\begin{array}{l}60 \mathrm{~min} \\
\text { resuscitation }\end{array}$ \\
\hline \multirow[t]{2}{*}{ Heart rate, $\min ^{-1}$} & DCLHb & $123 \pm 20$ & $127 \pm 17$ & $181 \pm 22 *$ & $147 \pm 14^{*}, \#$ & $138 \pm 20$ \\
\hline & HSA & $116 \pm 14$ & $118 \pm 15$ & $181 \pm 24^{*}$ & $155 \pm 15^{*}, \#$ & $149 \pm 16^{\#}$ \\
\hline \multirow[t]{2}{*}{$\mathrm{MAP}, \mathrm{mm} \mathrm{Hg}$} & DCLHb & $117 \pm 20$ & $124 \pm 18$ & $45 \pm 2 *$ & $113 \pm 13^{*, \S}$ & $115 \pm 30^{\S}$ \\
\hline & HSA & $114 \pm 20$ & $117 \pm 15$ & $46 \pm 4^{*}$ & $66 \pm 16^{*, \#}$ & $69 \pm 16^{\#}$ \\
\hline \multirow{2}{*}{$\begin{array}{l}\text { SVRI, } \\
\text { dyn } \cdot \mathrm{s} \cdot \mathrm{cm}^{-5} \cdot \mathrm{m}^{-2}\end{array}$} & DCLHb & $2,273 \pm 627$ & $2,430 \pm 657$ & $2,535 \pm 785$ & $2,271 \pm 450^{\S}$ & $2,650 \pm 833^{\S}$ \\
\hline & HSA & $1,848 \pm 505$ & $1,930 \pm 348$ & $2,136 \pm 656$ & $866 \pm 166^{*}, \#$ & $831 \pm 169^{\#}$ \\
\hline \multirow[t]{2}{*}{$\mathrm{CaO}_{2}, \mathrm{ml} \cdot \mathrm{dl}^{-1}$} & DCLHb & $13.2 \pm 1.7$ & $12.7 \pm 2.0$ & $11.6 \pm 1.3$ & $10.3 \pm 0.9^{*, \#, \S}$ & $9.3 \pm 1.2^{\#, \S}$ \\
\hline & HSA & $12.7 \pm 1.2$ & $12.7 \pm 1.5$ & $11.6 \pm 1.9$ & $6.8 \pm 1.0^{*} \#$ & $6.6 \pm 0.3^{\#}$ \\
\hline
\end{tabular}

Results and differences between DCLHb and HSA at the different measurement time points.

$\mathrm{MAP}=$ Mean arterial pressure; $\mathrm{SVRI}=$ systemic vascular resistance indexed to body surface area; $\mathrm{CaO}_{2}=$ arterial oxygen content [6] .

${ }^{*} \mathrm{p}<0.05$ vs. previous measurement; ${ }^{\#} \mathrm{p}<0.05$ vs. baseline; ${ }^{\S} \mathrm{p}<0.05$ DCLHb vs. HSA.

Table 3. LV diastolic function

\begin{tabular}{|c|c|c|c|c|c|c|}
\hline Parameter & Group & Baseline & $\begin{array}{l}\text { Critical } \\
\text { coronary stenosis }\end{array}$ & $\begin{array}{l}60 \mathrm{~min} \\
\text { shock }\end{array}$ & Resuscitation & $\begin{array}{l}60 \text { min } \\
\text { resuscitation }\end{array}$ \\
\hline \multirow[t]{2}{*}{$\mathrm{LVP}, \mathrm{mm} \mathrm{Hg}$} & DCLHb & $134 \pm 19$ & $137 \pm 21$ & $68 \pm 11^{*}$ & $120 \pm 26^{\S, *}$ & $125 \pm 47$ \\
\hline & HSA & $138 \pm 26$ & $136 \pm 16$ & $74 \pm 15^{*}$ & $91 \pm 29$ & $88 \pm 19^{\#}$ \\
\hline \multirow[t]{2}{*}{ LVEDP, mm Hg } & DCLHb & $6 \pm 2$ & $6 \pm 2$ & $3 \pm 3^{*}$ & $9 \pm 3^{*}$ & $9 \pm 4$ \\
\hline & HSA & $8 \pm 3$ & $8 \pm 3$ & $4 \pm 2 *$ & $17 \pm 8^{*, \#, \S}$ & $18 \pm 9^{\#, \S}$ \\
\hline \multirow{2}{*}{$\begin{array}{l}\mathrm{LVdp} / \mathrm{dt}_{\min }, \\
\mathrm{mm} \mathrm{Hg} \cdot \mathrm{s}^{-1}\end{array}$} & DCLHb & $-2,364 \pm 497$ & $-2,229 \pm 552$ & $-1,097 \pm 368^{*}$ & $-1,683 \pm 658^{*}$ & $-1,753 \pm 704$ \\
\hline & HSA & $-2,239 \pm 483$ & $-2,261 \pm 418$ & $-1,047 \pm 333^{*}$ & $-1,435 \pm 484^{\#}$ & $-1,337 \pm 248^{\#}$ \\
\hline \multirow[t]{2}{*}{$\tau, \mathrm{ms}$} & $\mathrm{DCLHb}$ & $28.8 \pm 7.9$ & $29.7 \pm 6.9$ & $20.9 \pm 10.5$ & $33.6 \pm 9.9^{*} \S$ & $30.8 \pm 8.2$ \\
\hline & HSA & $27.8 \pm 4.3$ & $27.1 \pm 6.9$ & $25.3 \pm 7.2$ & $22.9 \pm 5.4$ & $24.3 \pm 3.6$ \\
\hline \multirow[t]{2}{*}{$\mathrm{EDSL}_{\text {control }}, \mathrm{mm}$} & DCLHb & $11.9 \pm 2.5$ & $11.8 \pm 2.6$ & $10.7 \pm 2.5$ & $12.2 \pm 2.7$ & $11.9 \pm 2.7$ \\
\hline & HSA & $11.9 \pm 2.7$ & $12.1 \pm 3.1$ & $10.3 \pm 2.4$ & $12.1 \pm 3.3$ & $12.2 \pm 4.3$ \\
\hline \multirow[t]{2}{*}{ EDSL $_{\text {poststen }}, \mathrm{mm}$} & DCLHb & $13.3 \pm 3.5$ & $13.3 \pm 3.4$ & $12.2 \pm 3.4$ & $13.3 \pm 3.9$ & $13.4 \pm 3.6$ \\
\hline & HSA & $11.5 \pm 2.6$ & $11.3 \pm 2.6$ & $9.8 \pm 2.5$ & $11.9 \pm 2.9$ & $11.9 \pm 3.9$ \\
\hline
\end{tabular}

Results and differences between DCLHb and HSA at the different measurement time points.

LVP = Left ventricular pressure; LVEDP = left ventricular end-diastolic pressure;

$\mathrm{LVdp} / \mathrm{dt}_{\min }=$ maximum rate of left ventricular pressure decline; $\tau=$ time constant of diastolic relaxation;

$\mathrm{EDSL}_{\text {control }}$ and $\mathrm{EDSL}_{\text {poststen }}=$ end-diastolic myocardial segment length in normally perfused and poststenotic myocardium.

* $\mathrm{p}<0.05$ vs. previous measurement; ${ }^{*} \mathrm{p}<0.05$ vs. baseline; ${ }^{\S} \mathrm{p}<0.05$ DCLHb vs. HSA.

\section{Critical Coronary Stenosis}

Induction of critical LAD stenosis did not produce changes in the investigated parameters of LV diastolic properties within each animal. Neither at 'baseline' nor at 'critical coronary stenosis' were any significant differences between the groups established (table 2).

\section{Hemorrhagic Shock}

At ' 60 min shock', no differences between the groups were observed concerning the investigated parameters of LV diastolic function (table 3). LVP and LVEDP decreased by 49 and $50 \%$, respectively $(\mathrm{p}<0.05)$, while the time constant of LV diastolic relaxation ' $\tau$ ' as well as the 


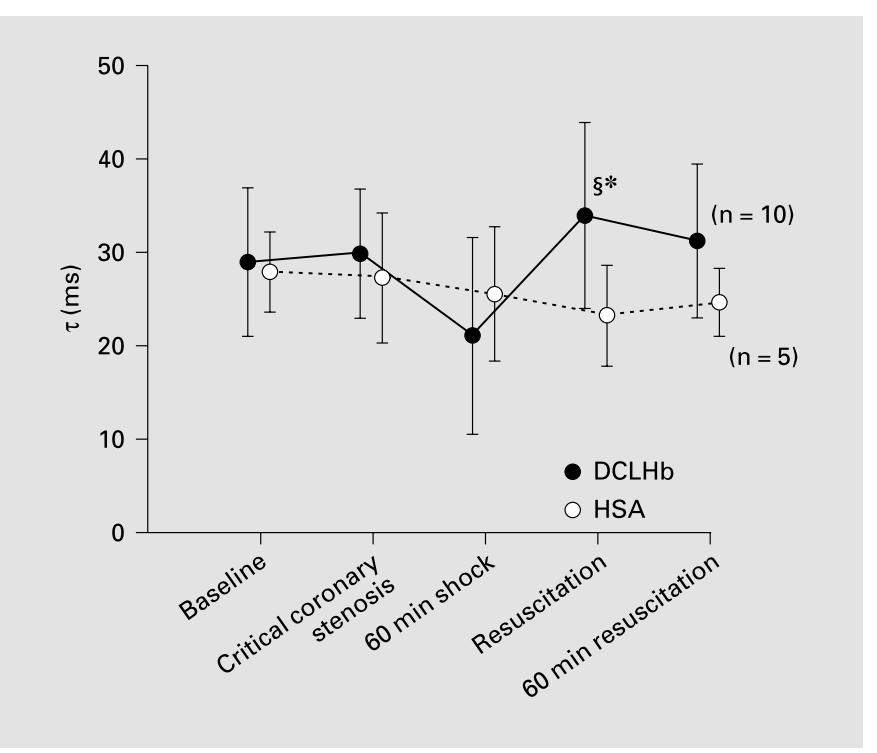

Fig. 2. Immediately after fluid resuscitation, the time constant of LV diastolic relaxation $(\tau)$ was prolonged in DCLHb-treated animals. $60 \mathrm{~min}$ after resuscitation, no significant differences between DCLHb group and HSA-survivors were observed. ${ }^{\S} \mathrm{p}<0.05 \mathrm{DCLHb}$ vs. HSA; $* \mathrm{p}<0.05$ vs. previous measurement.

end-diastolic segment length did not reveal significant alterations in the normally perfused or in the poststenotic myocardium. Maximal LV pressure decline $\left(\mathrm{LVdp} / \mathrm{dt}_{\min }\right)$ decreased by $52 \%(\mathrm{p}<0.05)$.

\section{Resuscitation}

The MAP was increased by both resuscitation modes, but MAP rose more pronounced in the DCLHb-group $(\mathrm{p}<0.05)$. Consistently, the systemic vascular resistance indexed to body surface area was significantly higher in DCLHb-treated animals immediately as well as $60 \mathrm{~min}$ after resuscitation.

The infusion of DCLHb enabled a permanent restoration of LVP and LVEDP $(p<0.05)$, while in HSA-treated animals the LVEDP was elevated immediately after resuscitation when compared with 'baseline' $(p<0.05)$. 60 min after resuscitation, even HSA survivors presented a significantly higher LVEDP than DCLHb-treated animals.

The end-diastolic myocardial segment length was not influenced in normally perfused or in poststenotic myocardia by either of the two treatments. $\mathrm{LVdp} / \mathrm{dt}_{\min } \mathrm{did}$ not show significant differences between the groups, but in contrast to HSA survivors, $\mathrm{LVdp} / \mathrm{dt}_{\min }$ was only restored to baseline values by infusion of DCLHb.
In contrast to $L V d p / d_{\min }, \tau$ was elevated after infusion of DCLHb with significantly higher values compared with 'baseline' as well as with HSA-treated animals. However, this difference vanished during the subsequent observation period, so that $60 \mathrm{~min}$ after resuscitation no differences to the 5 HSA survivors were observed (fig. 2, 3).

\section{Discussion}

The main finding of the study was the reduction of mortality from $50 \%$ within the HSA-treated control group to $0 \%$ after resuscitation with the HBOC. The crucial advantage of DCLHb compared with HSA was the superior myocardial oxygenation due to the augmentation of arterial $\mathrm{O}_{2}$ content and of CPP enabling a complete reversal of shock-induced subendocardial ischemia [6].

Therefore, we hypothesized the presence of significant differences between groups concerning the LV diastolic function which in turn is a sensitive marker of myocardial ischemia. However, the investigated parameters reflecting LV diastolic properties did not demonstrate superiority of the HBOC. Although only treatment with DCLHb restored $\mathrm{LVdp} / \mathrm{dt}_{\min }$ to baseline values, neither $\mathrm{LVdp} /$ $\mathrm{dt}_{\text {min }}$ nor end-diastolic myocardial segment length revealed significant differences between the DCLHb group and the 5 HSA survivors. Converse to our hypothesis, the time constant of LV diastolic relaxation ' $\tau$ ' was elevated over the baseline level immediately upon infusion of $\mathrm{DCLHb}$, indicating retardation of LV isovolumic relaxation.

At $\mathrm{RBC}$-free fluid resuscitation from hemorrhagic shock, the heart is responsible for an adequate compensatory hemodynamic response to hemodilution, while at the same time the cardiac function is endangered due to an imbalance between increased myocardial $\mathrm{O}_{2}$ demand and reduced $\mathrm{O}_{2}$ supply. As a consequence, acute myocardial failure becomes apparent [18]. The imbalance of myocardial oxygenation is aggravated when the myocardial blood flow a priori is compromised due to coronary stenosis. A critical stenosis $(\sim 75-85 \%)$ does not affect blood flow at rest, but completely deprives the vessel of its vasodilator

Fig. 3. Typical LV pressure/work diagrams of 1 DCLHb-treated animal (left) and 1 HSA survivor (right). Immediately after resuscitation, the asymptotic slope is right shifted in the DCLHb-treated ani$\mathrm{mal}$, indicating prolongation of the $\mathrm{LV}$ diastolic relaxation time constant $(\tau) .60 \mathrm{~min}$ after resuscitation, this difference has disappeared. 

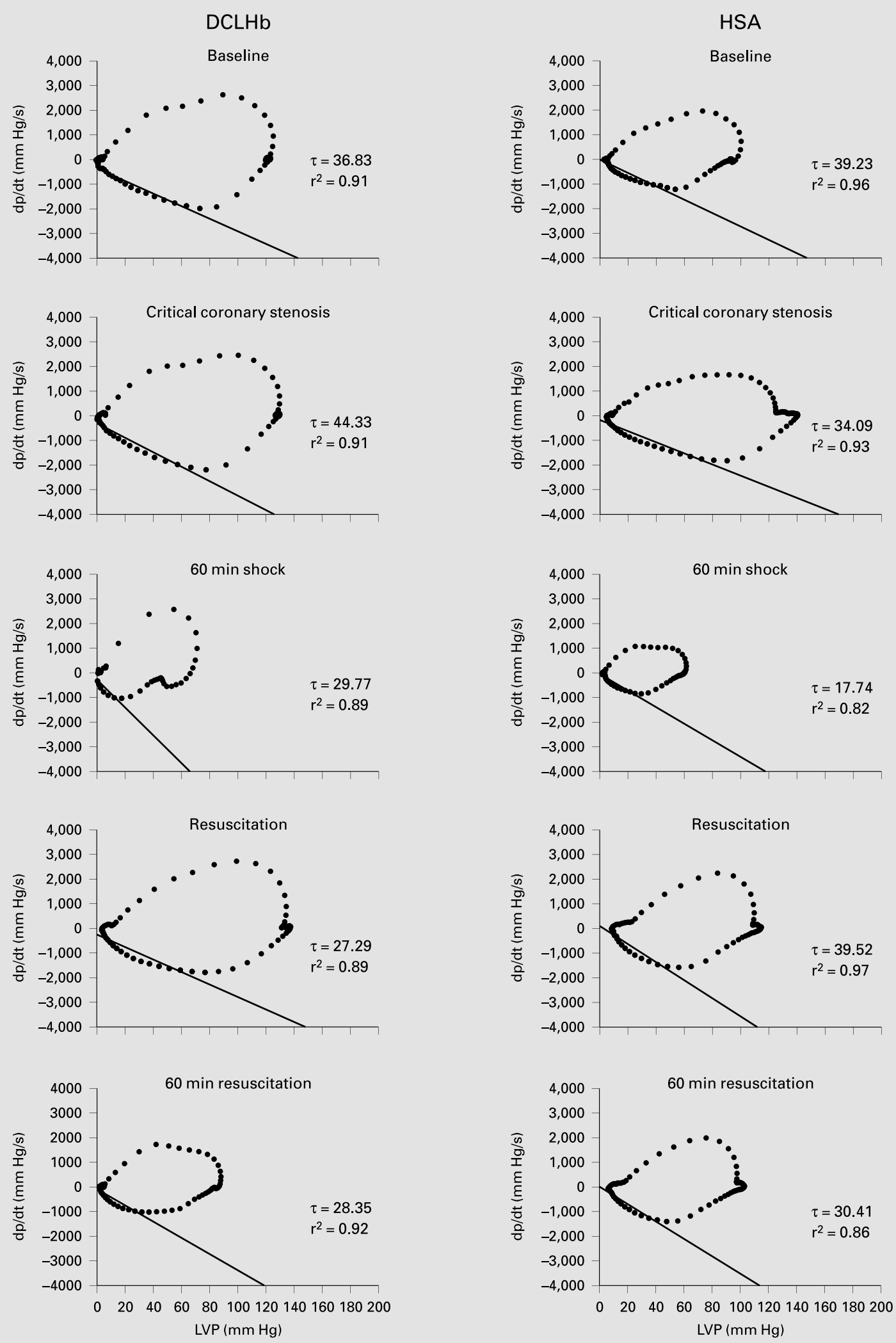

3 
reserve [19]. Hence, the compensatory increase of coronary blood flow during hemodilution cannot take place [12]. Thereby myocardial oxygenation and energy metabolism get impaired which directly affects isovolumic LV relaxation: as the transport of ionized calcium, which is released from its binding site on troponin $\mathrm{C}$ in order to allow the actin-myosin cross-bridges to dissociate, occurs against a large concentration gradient, high-energy phosphates are required for active myocardial relaxation [20, 21]. Thus, myocardial 'stiffness' and compromised LV diastolic function, as revealed by prolongation of ' $\tau$ ', are accepted to be consequences of insufficient myocardial oxygenation [4].

Although LV afterload was increased after infusion of DCLHb due to peripheral vasoconstriction, the myocardial oxygenation remained adequate [6]. Therefore, the question arises how the improved myocardial oxygenation fits with deteriorated LV diastolic function upon DCLHb infusion. Retardation of LV isovolumic relaxation, as shown by the prolongation of $\tau$ immediately upon infusion of DCLHb, is all the more remarkable, as even a period of $1 \mathrm{~h}$ of hemorrhagic shock in the presence of a critical coronary stenosis did not exert any significant changes of $\tau$.

If prolongation of $\tau$ indeed reflects inadequate myocardial oxygenation, it may be speculated that $\mathrm{O}_{2}$ transported by DCLHb was not be delivered to the myocardium due to either insufficient molecular $\mathrm{O}_{2}$ release or impairment of the myocardial perfusion as a result of coronary vasoconstriction.

However, our data demonstrate that $33 \%$ of the $\mathrm{O}_{2}$ consumed by the poststenotic myocardium was supplied by DCLHb [6] and that it is unlikely that the $\mathrm{O}_{2}$ transport is less effective to the myocardium with uncompromised perfusion. Moreover, the $\mathrm{O}_{2}$ affinity of DCLHb is lower when compared to native hemoglobin. The $\mathrm{P}_{50}$ value of $\mathrm{DCLHb}$ is higher, and the $\mathrm{O}_{2}$ dissociation curve is slightly shifted to the right as compared with native hemoglobin. This should allow DCLHb to release $\mathrm{O}_{2}$ more readily to the tissues [22].

In a previous study [23], we could demonstrate a direct vasoconstrictive effect of DCLHb on coronary and intramyocardial vessels, as the coronary vascular resistance was elevated after intended hemodilution with DCLHb. DCLHb-induced vasoconstriction might affect myocardial perfusion, leading to redistribution of myocardial blood flow, increased myocardial blood flow heterogeneity, and regional myocardial hypoperfusion. However, in the experimental model of the present study, we could also demonstrate that resuscitation from hemorrhagic shock with DCLHb tended to restore the normal distribution of regional myocardial blood flow [16].

There is no proof of inadequate myocardial oxygenation after infusion of DCLHb, but probably the alteration of ' $\tau$ ' upon hemodilution with DCLHb is caused by changes of macrohemodynamic parameters: normally, hemodilution increases LV preload by augmentation of venous return and decreases $L V$ afterload due to peripheral vasodilation. The diastolic function has been shown to remain unchanged during moderate acute isovolemic hemodilution (hematocrit 20\%) [24]. Besides, hemodilution with DCLHb seems to have contrary effects, as vasoconstriction after administration of DCLHb has been demonstrated to be more strongly pronounced in arteries than in veins [25]. Thereby, LV preload is reduced due to sequestration of the circulating blood volume in capacitance blood vessels, whereas LV afterload is increased due to nitric oxide scavenging.

Alterations of LV pre- and afterload (e.g., by administration of vasodilating and vasoconstrictive agents) have been shown to elicit shifts in the diastolic pressure-volume relation which has been explained by the influence of the pericardial pressure [26]. However, the pericardium was kept open throughout the whole protocol. Therefore, the end-diastolic myocardial segment length EDSL $_{\text {control }}$ and $\mathrm{EDSL}_{\text {poststen }}$ ), reflecting the LV end-diastolic volume, might be a parameter unsuitable for assessment of LV diastolic properties in this experimental setting.

Despite the open pericardium, the transmural LVP was significantly elevated by the increased afterload following infusion of DCLHb. Nevertheless, the peak velocity of the LVP decrease ( $\left.\mathrm{LVdp} / \mathrm{dt}_{\min }\right)$ did not indicate alterations of $\mathrm{LV}$ diastolic function. Yet, the $\mathrm{LVdp} / \mathrm{dt}_{\min }$ is highly dependent on LV pre- and afterload which were significantly influenced by DCLHb. Due to load dependence, the $\mathrm{LVdp} / \mathrm{dt}_{\min }$ might also be unreliable for the evaluation of LV diastolic function in this situation [27].

In contrast, ' $\tau$ ' is a preload-independent measure of isovolumic relaxation [28]. Variations of LV afterload do, however, significantly correlate with alterations of the isovolumic relaxation time [29, 30].

Deterioration of the LV diastolic function immediately upon resuscitation with DCLHb seems to be predominantly the result of vasoconstriction. Therefore, our hypothesis of a DCLHb-improved LV diastolic function after resuscitation has to be rejected.

However, the positive effects of the vasoconstrictive and $\mathrm{O}_{2}$-transporting properties of DCLHb on global myocardial oxygenation enabled adequate myocardial $\mathrm{O}_{2}$ supply and thereby complete reversal of subendocardial isch- 
emia. Although DCLHb obviously failed to restore the $\mathrm{LV}$ diastolic function, the beneficial effects on myocardial oxygenation clearly predominate, resulting in a significant reduction of the mortality [6].

Despite repeatedly proven superiority of DCLHb in preclinical models, a clinical phase III study conducted at 18 US trauma centers indicated a significantly higher mortality in DCLHb-treated patients. After an interim data analysis, patient enrollment was suspended, as it seemed unlikely to meet the expected end points for efficacy and safety [31].

In September 1998, Baxter Healthcare terminated further clinical investigation in DCLHb. Nevertheless, investigation and development of new HBOC for operative and emergency medicine remains an issue of major interest.

\section{Acknowledgements}

The authors thankfully appreciate Mrs. A. Schropp's valuable technical assistance and Mr. O. Frisch and his team's excellent performance of animal care as well as Dr. A. Brederode and Prof. Dr. Dr. h.c. mult. K. Messmer for reviewing the manuscript.

\section{Appendix}

\section{Calculations}

The body surface area (BSA in $\mathrm{m}^{2}$ ) was calculated according to Holt et al. [32]:

$$
\mathrm{BSA}=\mathrm{k} \cdot \mathrm{BW}^{2 / 3}\left(\mathrm{~m}^{2}\right)
$$

where $\mathrm{BW}=$ body weight (in $\mathrm{kg}$ ) and $\mathrm{k}=9$.

The systemic vascular resistance index (SVRI) was calculated as:

$$
\mathrm{SVRI}=\frac{(\mathrm{MAP}-\mathrm{CVP}) \cdot 79.9}{\mathrm{CI}}\left(\frac{\mathrm{dyn} \cdot \mathrm{s}}{\mathrm{cm}^{5} \cdot \mathrm{m}^{2}}\right)
$$

The arterial oxygen content $\left(\mathrm{CaO}_{2}\right)$ was calculated as:

$$
\begin{aligned}
& \mathrm{CaO}_{2}=\left(\left[\mathrm{Hb}_{\text {porcine }}\right] \cdot \mathrm{SaO}_{2 \text { porcine }} \cdot 1.34\right)+ \\
& \left([\mathrm{DCLHb}] \cdot \mathrm{SaO}_{2 \mathrm{DLCHb}} \cdot 1.39\right)+\mathrm{paO}_{2} \cdot 0.0031
\end{aligned}
$$

\section{Anesthetics}

For induction and maintenance of anesthesia, the following drugs were applied: ketamine (Ketavet ${ }^{\mathrm{TM}}$; Parke-Davis, Berlin, Germany), midazolam (Dormicum ${ }^{\mathrm{TM}}$; Roche, Grenzach, Germany), atropine (Atropinsulfat ${ }^{\mathrm{TM}}$; Braun, Melsungen, Germany), methohexital (Brevimytal $^{\mathrm{TM}}$; Lilly, Bad Homburg, Germany), fentanyl (Fentanyl ${ }^{\mathrm{TM}}$; Janssen, Neuss, Germany), and pancuronium (Pancuronium ${ }^{\mathrm{TM}}$; $\mathrm{Cu}-$ ramed, Karlsruhe, Germany).

\section{References}

1 Peitzman AB: Hemorrhagic shock. Curr Probl Surg 1995;32:925-1002.

2 Ledingham IM: Heart failure in experimental refractory shock. Eur J Intensive Care Med 1976;2:111-117.

3 Alyono D, Ring WS, Anderson RW: The effects of hemorrhagic shock on the diastolic properties of the left ventricle in the conscious dog. Surgery 1978;83:691-698.

4 Miyaji K, Sugiura S, Inaba H, Takamoto S, Omata S: Myocardial tactile stiffness during acute reduction of coronary blood flow. Ann Thorac Surg 1978;69:151-155.

5 Welte M, Lackermeier P, Habler O, Kleen M, Kemming G, Frey L, Zwissler B, Messmer K: Effect of hypertonic saline/dextran on post-stenotic myocardial perfusion, metabolism and function during resuscitation from hemorrhagic shock in anaesthetized pigs. Shock 1997;7: 119-130.

6 Habler O, Kleen M, Pape A, Meisner F, Kemming G, Messmer K: Diaspirin cross-linked hemoglobin (DCLHb) reduces mortality of severe hemorrhagic shock in pigs with critical coronary stenosis. Crit Care Med 2000;28: 1889-1898.

7 Habler O, Kleen M, Messmer K: Künstliche Sauerstoffträger - Alternativen zur Fremdbluttransfusion? Zentralbl Chir 1999;124:260270.

DCLHb and Left Ventricular Diastolic

Function
8 Habler O, Messmer K: Artificial oxygen carriers. Baillière's Clin Anaesthesiol 1997;11: 289-300.

9 Ketcham EM, Cairns CB: Hemoglobin-based oxygen carriers: development and clinical potential. Ann Emerg Med 1999;33:326-337.

10 Doherty DH, Doyle MP, Curry SR, Vali RJ, Fattor TJ, Olson JS, Lemon DD: Rate of reaction with nitric oxide determines the hypertensive effect of cell-free hemoglobin. Nat Biotechnol 1998;16:672-676.

11 Alayash AI: Hemoglobin-based blood substitutes: Oxygen carriers, pressor agents, or oxidants? Nat Biotechnol 1999;17:545-549.

12 Levy PS, Kim SJ, Eckel PK, Chavez R, Ismail EF, Gould SA, Salem MR, Crystal GJ: Limit to cardiac compensation during acute isovolemic hemodilution: Influence of coronary stenosis. Am J Physiol 1993;265:H340-H349.

13 Gould KL, Lipscomb K, Hamilton GW: Physiologic basis for assessing critical coronary stenosis. Am J Cardiol 1974;33:87-94.

14 Jesch F, Kloevekorn WP, Sunder-Plassmann L, Messmer K: Importance of uptake in experimental hemorrhagic shock. Res Exp Med (Berl) 1972;157:267-269.

15 Heymann MA, Payne BD, Hofmann JIE, Rudolph AM: Blood flow measurements with radionuclide-labeled particles. Prog Cardiovasc Dis 1977;20:55-79.
16 Kleen M, Habler O, Meisner F, Kemming G, Pape A, Messmer K: Effects of primary resuscitation from shock on distribution of myocardial blood flow. J Appl Physiol 2000;88:373385.

17 Beattie C, Humphrey LS, Maruschak G: Determination of decay constants from time-varying data. Anesthesiology 1989;70:159-160.

18 Sarnoff SJ, Case RB, Waithe PE, Isaacs JP: Insufficient coronary flow and myocardial failure as a complicating factor in hemorrhagic shock. Am J Physiol 1954;176:439-444.

19 Schad H, Heimisch W, Mendler N: Models of coronary artery disease: 'Critical' versus 'functional' coronary artery stenosis. Thorac Cardiovasc Surg 1991;39:13-18.

20 Eisenberg E, Hill TL: Muscle contraction and free energy transduction in biological systems. Science 1985;227:999-1006.

21 Litwin SE, Grossman W: Diastolic dysfunction as a cause of heart failure. J Am Coll Cardiol 1993;22(4 suppl A):49-55.

22 Baron JF: Hemoglobin therapy in clinical practice: Use and characteristics of DCLHb. Br J Anaesth 1998:81(suppl 1):34-37.

23 Meisner F, Kemming G, Habler O, Kleen M, Tillmanns J, Hutter J, Bottino DA, Thein E, Meier J, Wojtczyk C, Pape A, Messmer K: Diaspirin cross-linked hemoglobin enables extreme hemodilution beyond the critical hematocrit. Crit Care Med 2001;29:829-838. 
24 Habler O, Kleen M, Podtschaske A, Hutter J, Tiede M, Kemming G, Messmer K: Akute normovolämische Hämodilution (ANH). Effekte der ANH auf die diastolische Funktion des linken Ventrikels. Anästhesist 2000;49:939_ 948.

25 Freas W, Llave R, Jing M, Hart J, McQuillan $\mathrm{P}$, Muldoon S: Contractile effects of diaspirin cross-linked hemoglobin (DCLHb) on isolated porcine blood vessels. J Lab Clin Med 1995; 125:762-767.

26 Smiseth OA, Kingma I, Refsum H, Smith ER, Tyberg JV: The pericardial hypothesis: A mechanism of acute shifts of the left ventricular diastolic pressure-volume relation. Clin Physiol 1985;5:403-415.
27 Lew WYW: Evaluation of left ventricular diastolic function. Circulation 1989;79:13931397.

28 Varma SK, Owen RM, Smucker ML, Feldman MD: Is tau a preload-independent measure of isovolumetric relaxation? Circulation 1989;80: 1757-1765.

29 Langer SF, Schmidt HD: Different left ventricular relaxation parameters in isolated working rat and guinea pig hearts: Influence of preload, afterload, temperature and isoprenaline. Int $\mathbf{J}$ Card Imaging 1998;14:229-240.
30 Thomas JD, Flachskampf FA, Chen C, Guerrero JL, Picard MH, Levine RA, Weyman AE: Isovolumic relaxation time varies predictably with its time constant and aortic and left atrial pressures: Implications for the noninvasive evaluation of ventricular relaxation. Am Heart J 1992;124:1305-1313.

31 Sloan EP, Koenigsberg M, Gens D, Cipolle M, Runge J, Mallory MN, Rodman G: Diaspirin cross-linked hemoglobin (DCLHb) in the treatment of severe traumatic hemorrhagic shock. JAMA 1999;282:1857-1864.

32 Holt JP, Rhode EA, Kines H: Ventricular volumes and body weight in mammals. Am J Physiol 1968;215:704-715. 